\title{
The Construction and Comprehensive Analysis of a ceRNA Immunoregulatory Network and Tissue-Infiltrating Immune Cells in Atrial Fibrillation
}

\author{
Xing Liu (D)' \\ Guoqiang Zhong $\mathbb{D}^{2}$ \\ Wenbin $\mathrm{Li}\left(\mathbb{D}^{\prime}\right.$ \\ Yiqian Zeng $\mathbb{D}^{3}$ \\ Mingxing $\mathrm{Wu} \mathbb{D}^{1}$ \\ 'Department of Cardiology, Xiangtan \\ Central Hospital, Xiangtan, Hunan, \\ People's Republic of China; ${ }^{2}$ Department \\ of Cardiology, Guangxi Cardiovascular \\ Institute, The First Affiliated Hospital of \\ Guangxi Medical University, Guangxi, \\ People's Republic of China; ${ }^{3}$ Department \\ of Critical Care Medicine, Zhuzhou \\ Central Hospital, Zhuzhou, Hunan, \\ People's Republic of China
}

Correspondence: Yiqian Zeng Department of Critical Care Medicine, Zhuzhou Central Hospital, Zhuzhou, Hunan, People's Republic of China Email zengyiqian@stu.gxmu.edu.cn

Mingxing $W_{u}$

Department of Cardiology, Xiangtan

Central Hospital, Xiangtan, Hunan,

People's Republic of China

Email wmx917I2I@I26.com
Background: At present, the mechanisms behind atrial fibrillation (AF) pathogenesis are still unclear. We construct a ceRNA immunoregulatory network to further understand the mechanism of AF.

Methods: Four AF mRNA datasets from the Gene Expression Omnibus (GEO) database were integrated by SVA method. AF-related immune genes (AF-IRGs) were selected via combining ImmPort database with the genes in the module most associated with AF obtained by a weighted gene coexpression network analysis (WGCNA). Then, circRNA and miRNA expressions from the GEO database were extracted and mapped with related databases. Next, an immune-related circRNA-miRNA-mRNA ceRNA network was constructed and hub genes were filtered from a protein-protein interaction (PPI) network, and the differentially expressed (DE) hub genes in AF were further screened. Additionally, immune infiltration was investigated in AF by using CIBERSORT. Subsequently, the relationships between DE hub genes and AF-related infiltrating immune cells were performed by using Pearson correlation coefficients. Ulteriorly, the immune-cells-related ceRNA subnetwork in AF was built.

Results: A total of 95 AF-IRGs were detected, and an immune-related ceRNA network in AF was constructed with 12 circRNAs, 7 miRNAs and 50 mRNAs. The immune infiltration analysis indicated that a higher level of neutrophils, as well as a lower level of $\mathrm{T}$ cells regulatory (Tregs) and NK cells activated in AF. Four DE hub genes (CXCL12, IL7R, TNFSF13B, CD8A) were associated with Tregs or NK cells activated immune cells $(\mathrm{P}<$ 0.05 ). Tregs or NK cells activated immune cells-related ceRNA subnetwork including 5 circRNAs (has_circ_0001190, has_circ_0006725, has_circ_0079284, has_circ_0005299, and has_circ_0002103), 4 miRNAs (has-miR-198, has-miR-623, has-miR-1246, and has-miR -339-3p) and $4 \mathrm{DE}$ hub genes was eventually constructed in AF.

Conclusion: Our results provide new insights into the molecular mechanisms governing AF progression from the perspective of immune-related ceRNA network.

Keywords: atrial fibrillation, competitive endogenous RNA, immune infiltration

\section{Introduction}

Atrial fibrillation (AF) is the most frequently occurring type of arrhythmia and its incidence increases with age. In addition, AF is closely associated with poor quality of life, embolic stroke, heart failure, and elevated mortality. ${ }^{1}$ However, the underlying mechanisms of AF remain unclear. Understanding the mechanisms could help us to find new strategies for treating AF. Therefore, it is necessary to add our further insights into cellular and biological mechanisms of AF and to evolve more effective treatments for AF. 
At present, the mechanisms behind AF pathogenesis are mainly reflected in atrial electrical and structural remodeling. ${ }^{2}$ Furthermore, research have shown that a link between inflammation and atrial fibrosis, and inflammation and its associated immune response may play a critical role in the occurrence and maintenance of $\mathrm{AF}^{3,4}$ Study found that $\mathrm{C}$-reactive protein (CRP) was independently associated with the occurrence of $\mathrm{AF}$, and the incidence of $\mathrm{AF}$ increased with the increase of CRP expression. ${ }^{5}$ In addition, several immune-related genes (IRGs), such as C-X-C motif chemokine receptor 2 (CXCR2) and C-X-C motif chemokine 10 (CXCL10), may be implicated in the structural remodeling of $\mathrm{AF}^{6,7}$ The infiltration of immune cells has also been shown to influence the development of AF. For example, monocyte activation especially the enhanced migration ability plays a vital role in the pathogenesis of progression in atrial remodeling in $\mathrm{AF}$ patients. ${ }^{8}$

The role of the competing endogenous RNA (ceRNA) network has attracted much attention in recent years. ${ }^{9}$ As a novel type molecule with special biological functions, circRNAs can be used as microRNA (miRNA) sponge to competitively bind miRNAs, modulate alternative splicing, and regulate the expression of parental genes. ${ }^{10,11}$ Emerging data have elucidated that circRNA-miRNA-mRNA regulatory axes are implicated in the pathological progression of cardiovascular disease. For example, ciRS-7 can be used as miRNA-7a sponge to inhibit the expression of PARP and SP1, resulting in promoting myocardial apoptosis. ${ }^{12}$ CircRNA_000203 can enhance the expression of fibrosisassociated genes (Colla1 and CTGF) by suppressing targets of miR-26b-5p, leading to cardiac fibrosis, which is associated with AF. ${ }^{13}$ Therefore, circRNA-miRNA-mRNA regulatory network can deliver novel insights into the pathophysiology of AF. However, few studies have explored the relationship between immune infiltration and an immunerelated circRNA-miRNA-mRNA ceRNA network in AF.

In the present study, we provided new insights into the molecular mechanism of ceRNA immunoregulation during the progression of AF. Firstly, we identified AF-related immune genes (AF-IRGs) via combining ImmPort database with the genes in the module most associated with AF obtained by a weighted gene coexpression network analysis (WGCNA). Then, we constructed an immune-related circRNA-miRNA-mRNA ceRNA network based on circRNA-miRNA pairs and miRNA-mRNA pairs, and filtered differentially expressed (DE) hub genes from a protein-protein interaction (PPI) network. In addition, we performed an immune cell infiltration analysis in $\mathrm{AF}$ by using CIBERSORT. ${ }^{14}$ Finally, we selected DE hub genes associated with AF-related infiltrating immune cells by Pearson correlation coefficients to construct an immune-cells-related ceRNA subnetwork. Our study flowchart is shown in Figure 1.

\section{Methods}

\section{Data Acquisition and Processing}

One circRNA microarray dataset $\left(\mathrm{GSE} 129409^{15}\right)$, one miRNA microarray dataset $\left(\mathrm{GSE} 70887^{16}\right)$ and four mRNA microarray datasets (GSE41177, ${ }^{17}$ GSE79768, ${ }^{18}$ GSE31821, ${ }^{19}$ and GSE14975 ${ }^{20}$ ) were downloaded from gene expression omnibus (GEO, http://www.ncbi.nlm.nih. gov/geo/). ${ }^{21}$ All samples are from atrial tissue and the samples are classified as persistent AF and SR control groups; GSE129409 and GSE70887 were obtained from GPL21825 Arraystar Human circRNA Array V2, and GPL19546 Agilent-021827 Human miRNA Microarray respectively. Four mRNA datasets were generated using GPL570 (HGU133_Plus_2) Affymetrix Human Genome U133 Plus 2.0 Array. The GSE129409 contained left atrial appendage tissue from 3 persistent AF patients and 3 sinus rhythm (SR) controls. The GSE70887 contained atrial appendage tissue from 4 persistent AF patients and 2 SR controls. The GSE41177, GSE79768, GSE31821 and GSE14975 contained left atrial appendage tissue from 16 persistent AF patients and $3 \mathrm{SR}$ controls, 7 persistent AF patients and 6 SR controls, 2 persistent $\mathrm{AF}$ patients and $2 \mathrm{SR}$ controls, and 5 persistent $\mathrm{AF}$ patients and 5 SR controls, respectively.

The probe IDs were converted to symbol codes by using ActivePerl 5.24.2 software (https://www.activestate. com/products/perl/) to process series matrix files. The GSE14975 was log base 2 transformed by affy in R. ${ }^{22}$ After merging mRNA microarray data, the combat function in the sva package was used to eliminate batch effects and other unwanted variation between four mRNA datasets. $^{23}$ Finally, expression values were normalized using the "normalizeBetweenArrays" function of the package of "limma" in R software. ${ }^{24}$

The study protocol was approved by the Ethics Committee of Xiangtan central Hospital.

\section{Identification of Differential Expression of circRNAs, miRNAs and mRNAs}

The Limma package was utilized to screen DE circRNAs, DE miRNAs and DE mRNAs between SR samples and AF 


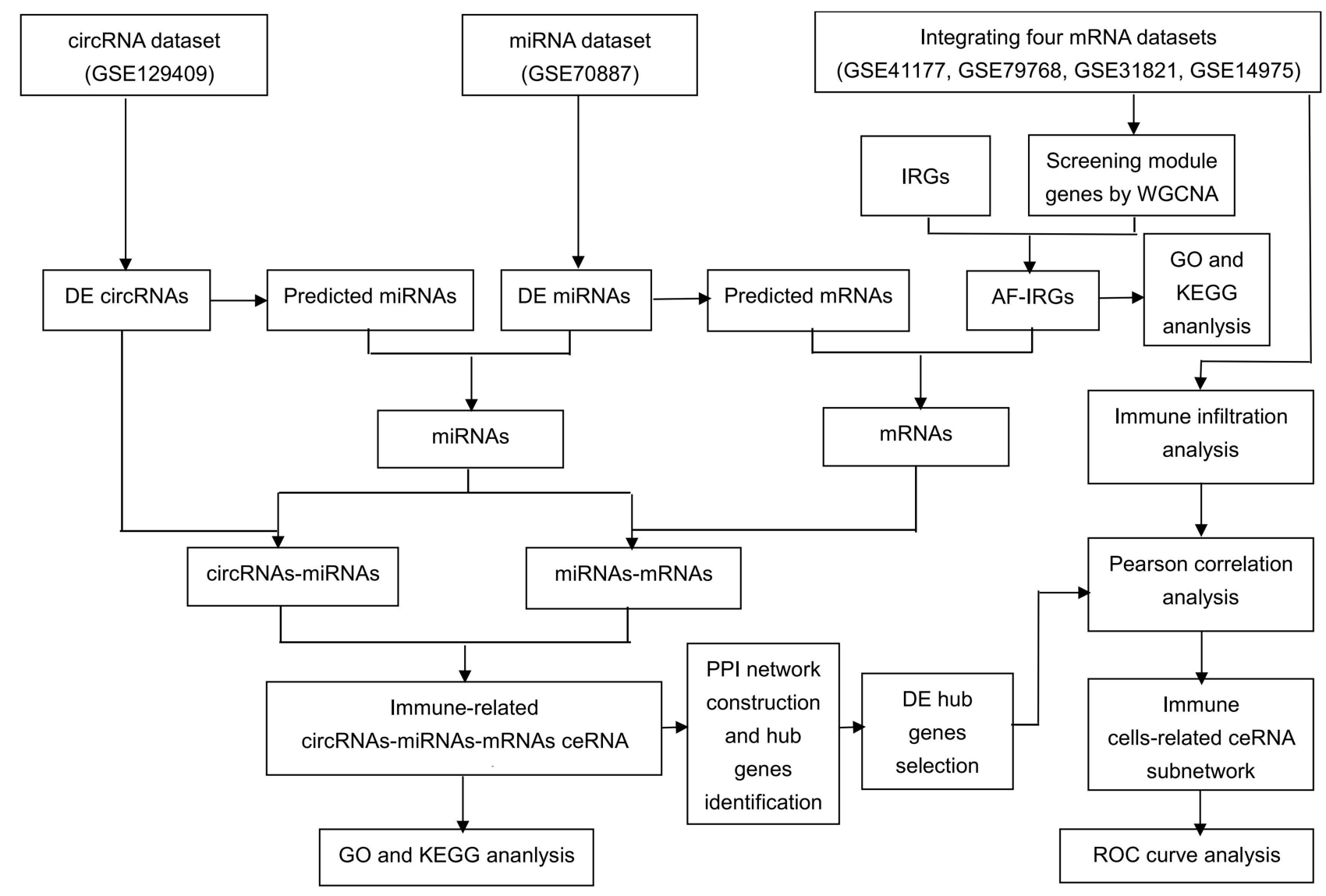

Figure I Flow diagram of study.

Abbreviations: DE, differentially expressed; IRGs, immune-related genes.

samples. $^{25}$ The GSE129409 was analyzed with $\left|\log _{2} \mathrm{FC}\right|>3$ and p.value $<0.05$ set as the cut-off point for the selection of DE circRNAs. To assess DE miRNAs in AF, GSE70887 was used, and transcripts with a cut-off point of $|\log 2 \mathrm{FC}|>1$ and p.value $<0.05$ were retrieved. The integrated dataset was analyzed with p.value $<0.05$ set as the cut-off point for the selection of DE mRNA. Heatmaps and volcano maps for DE circRNAs and DE miRNAs were created by using the "pheatmap" and "ggplot2" package of R software, respectively.

\section{Weighted Gene Co-Expression Network Analysis (WGCNA) Construction and Identification of AF-IRGs}

To identify candidate biomarkers and therapeutic targets, a system biology approach, WGCNA was used in gene coexpression network construction. In our study, the mRNA integrated dataset was constructed to gene co-expression network using the WGCNA package in $\mathrm{R}^{26}$ The soft thresholding power was set as 4 ; then, analyses of module-trait correlations, gene significance (GS), and module membership (MM) were performed. Statistically significant modules were defined as $\mathrm{P}<0.05$.

AF-IRGs were screened by matching 1793 IRGs to genes in the most relevant module with AF using the IRG dataset from the ImmPort database (https://www.imm port.org/shared/genelists).

\section{Construction of an Immune-Related circRNA-miRNA-mRNA ceRNA Network}

Information about circRNAs can be obtained in circBase (http://www.circbase.org/). ${ }^{27}$ Target miRNAs were predicted by the cancer-specific circRNA database (CSCD, https://gb. whu.edu.cn/CSCD/). ${ }^{28}$ For each DE circRNA, all predicted miRNAs were obtained. Next, miRNAs that overlapped with predicted and DE miRNAs were gathered. Then, the TargetScan database (http://www.targetscan.org/vert 72/) was used to predict the miRNA-targeted mRNAs. ${ }^{29}$ To this end, mRNAs recognized by TargetScan database were regarded as candidate targets and were intersected with AF- 
IRGs. Finally, an immune-related ceRNAs regulatory network in AF was constructed based on circRNA-miRNA pairs and miRNA-mRNA pairs. The ceRNA network was visualized by Cytoscape version 3.8.0 (https://cytoscape.org/).

\section{Enrichment Analysis}

$\mathrm{GO}$ and KEGG functional enrichment analysis of AFIRGs, and genes in the immune-related ceRNA network, respectively, were performed using the "clusterprofiler", "org.Hs.eg.db", “enrichplot", "ggplot2 Goplot" and "digest" packages of R/Bioconductor. p.value $<0.05$ was regarded as a statistically significant difference.

\section{Construction of PPI Regulatory Network and Identification of DE Hub Genes}

A PPI network of these genes in the immune-related ceRNA network was established by using the STRING database (http://string-db.org), ${ }^{30}$ and minimum interaction score $\geq 0.4$ was defined as the cut-off value. The PPI network was visualized by the CytoCope 3.8.0 software. In addition, top 10 hub genes in the PPI network were filtered by using the MCC algorithm of the Cytoscape plug-in cytoHubba, and DE hub genes were screened by intersection with the DE mRNAs.

\section{Immune Cell Infiltration Analysis}

To quantify the relative proportions of infiltrating immune cells in AF, CIBERSORT in R, was adopted to analyse the integrated mRNA dataset and calculate immune cell infiltrations. $\mathrm{P}<0.05$ was used to filter the samples. Principal component analysis (PCA) cluster plot of immune cell infiltration in AF and SR tissues was carried out by the "ggplot2", "FactoMineR", and "factoextra" package. Spearman correlation analysis using $\mathrm{R}$ software was applied to the 22 types of infiltrating immune cells, and a correlation heatmap was performed using the "Corrplot" package to visualize the result. The "vioplot" package was used to compare and visualize the levels of 22 immune cells between the AF and SR samples.

\section{Correlation Analysis Between DE Hub Genes and AF-Related Infiltrating Immune Cells}

The relationship between DE hub genes and AF-related infiltrating immune cells was performed by using Pearson correlation coefficients, which was visualized by using the "ggpubr" package of R.

\section{Construction of the Immune Cells-Related ceRNA Subnetwork}

After obtaining the DE hub genes related to AF-related immune cells by Pearson's method $(\mathrm{P}<0.05)$, we reconstructed an immune-cells-related ceRNA subnetwork from the immune-related circRNA-miRNA-mRNA ceRNA network and visualized it through the CytoCope 3.8.0 software. The boxplot maps of cicrRNAs, miRNAs and mRNAs expression levels in the subnetwork in their respective datasets were created by using the "ggpubr" and "reshape2" package of R software.

\section{Diagnostic Analysis of mRNAs of ceRNA Subnetwork for AF}

Receiver operator characteristic (ROC) curve analysis was performed using the "pROC" package of R to determine the effectiveness of these genes of ceRNA subnetwork in predicting AF.

\section{Results}

\section{Identification of DE circRNAs and DE miRNAs in AF}

In total, 103 DE circRNAs (59 up-regulated and 44 downregulated circRNAs) were identified in the GSE129409 dataset (Figure 2A and B). In addition, 16 up-regulated and 13 down-regulated miRNAs were screened in the GSE70887 dataset (Figure 2C and D).

\section{Coexpression Module Construction and AF-Related IRGs Screening}

In order to find the functional clusters in AF patients, gene co-expression networks were constructed from the integrated mRNA dataset with the WGCNA package. After setting the power as 4, 9 modules were excavated (Figure 3A). The results of the module-trait relationships are presented in Figure 3B, revealing that the red module was the most relevant modules with $\mathrm{AF}(\mathrm{r}=$ $0.45 ; \mathrm{P}=0.002$ ), including 280 genes. As GS and MM exhibit a very significant correlation, this finding implies that the genes in the red module tend to be highly correlated with AF (cor $=0.5, \quad \mathrm{P}=4.1 \mathrm{e}-19)$ (Figure 3C). In addition, the intersection of 1793 IRGs from the ImmPort database and the genes in red module was AF-related IRGs (AF-IRGs) (Figure 3D), including 95 genes. 
A

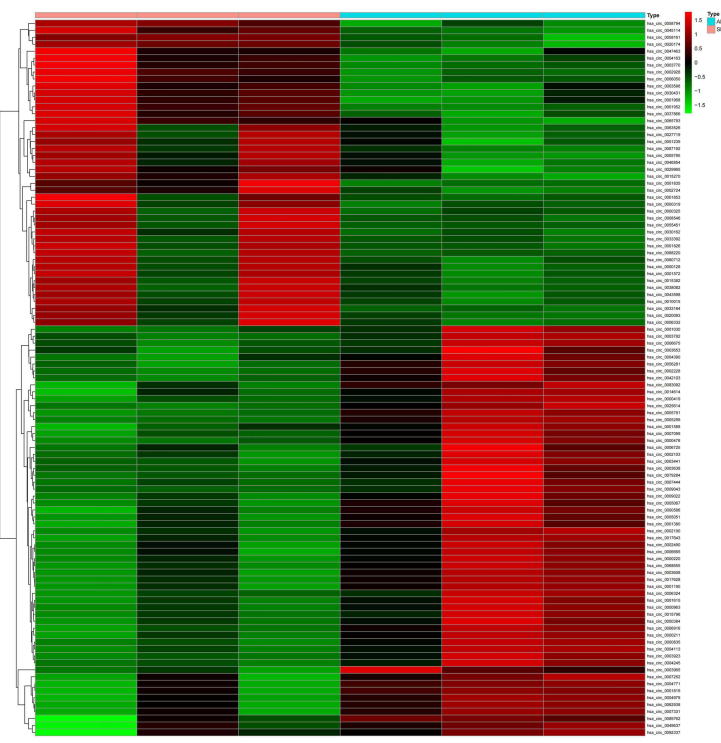

C

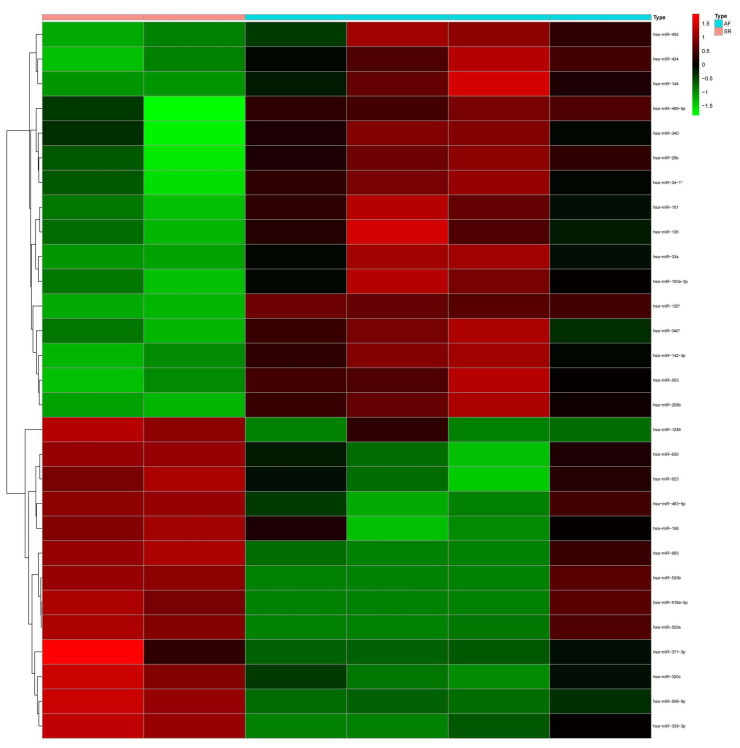

B

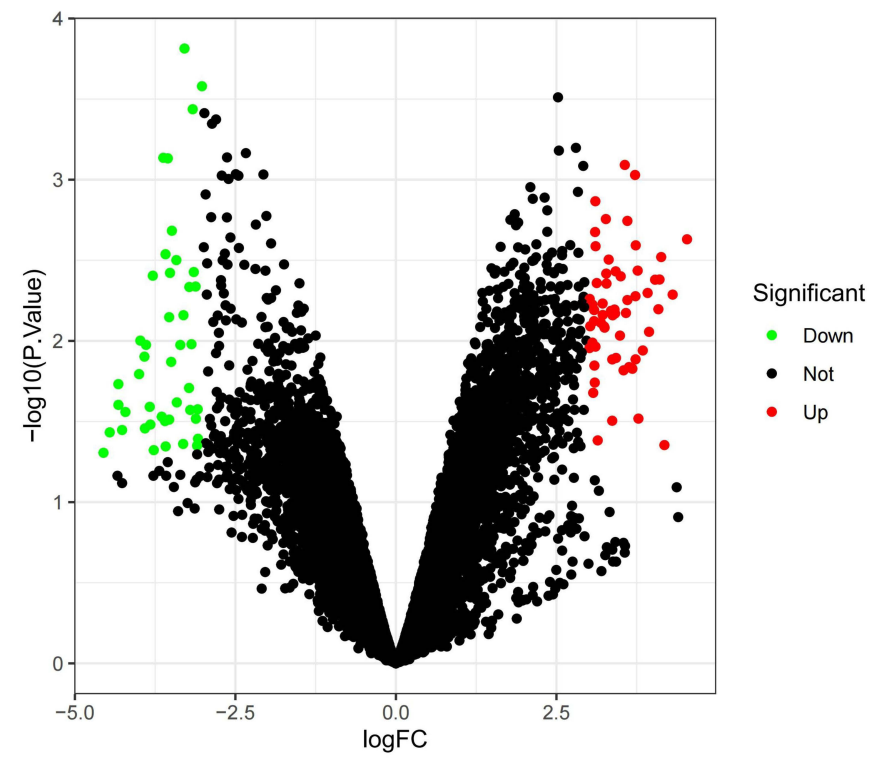

D

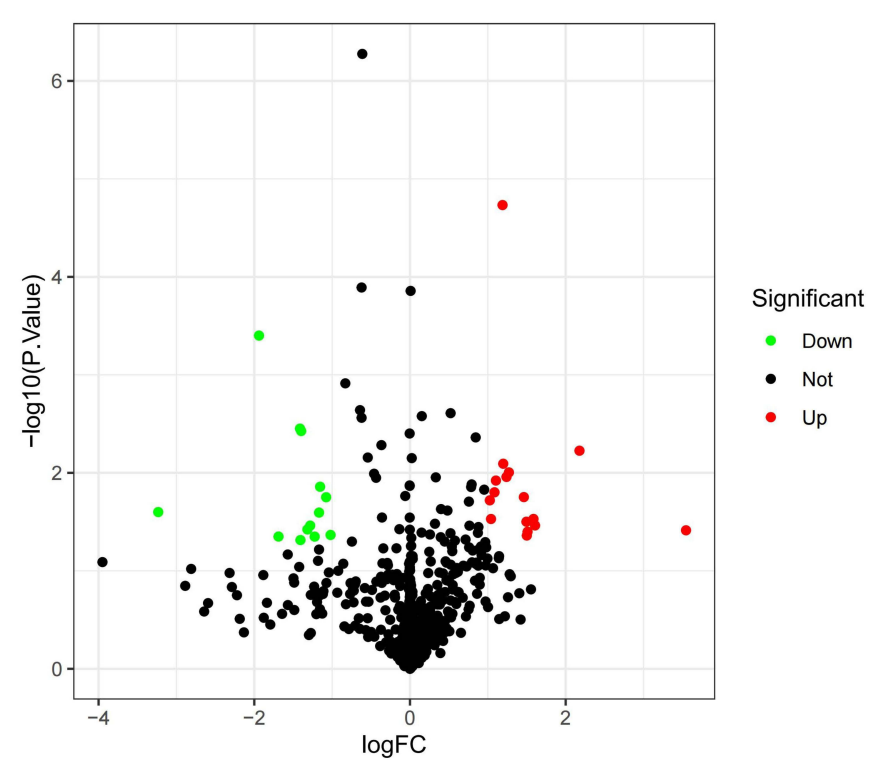

Figure 2 Identification of DE circRNAs, DE miRNAs in AF. Heat map (A) and volcano plots (B) of DE circRNAs in the GSE 29409 dataset. Heat map (C) and volcano plots (D) of DE miRNAs in the GSE70887 dataset.

Abbreviations: $A F$, atrial fibrillation; $D E$, differentially expressed.

\section{Enrichment Analysis of AF-IRGs}

The results of the GO analysis showed that the AF-IRGs were predominantly enriched in neutrophil chemotaxis (biological processes, BP), external side of plasma membrane (cellular components, $\mathrm{CC}$ ), and immune receptor activity and chemokine activity (molecular functions, MFs) (Figure 4A). KEGG pathway analysis showed significant enrichment in the viral protein interaction with cytokine and cytokine receptor, cytokine and cytokine receptor interaction and chemokine signaling pathway (Figure 4B).

\section{Construction of an Immune-Related circRNA-miRNA-mRNA ceRNA Network in AF}

Among the 103 DE circRNAs, 15 circRNAs were not searched in the CSCD database. We predicted that the remaining 88 circRNAs might target 1995 miRNAs by 
A

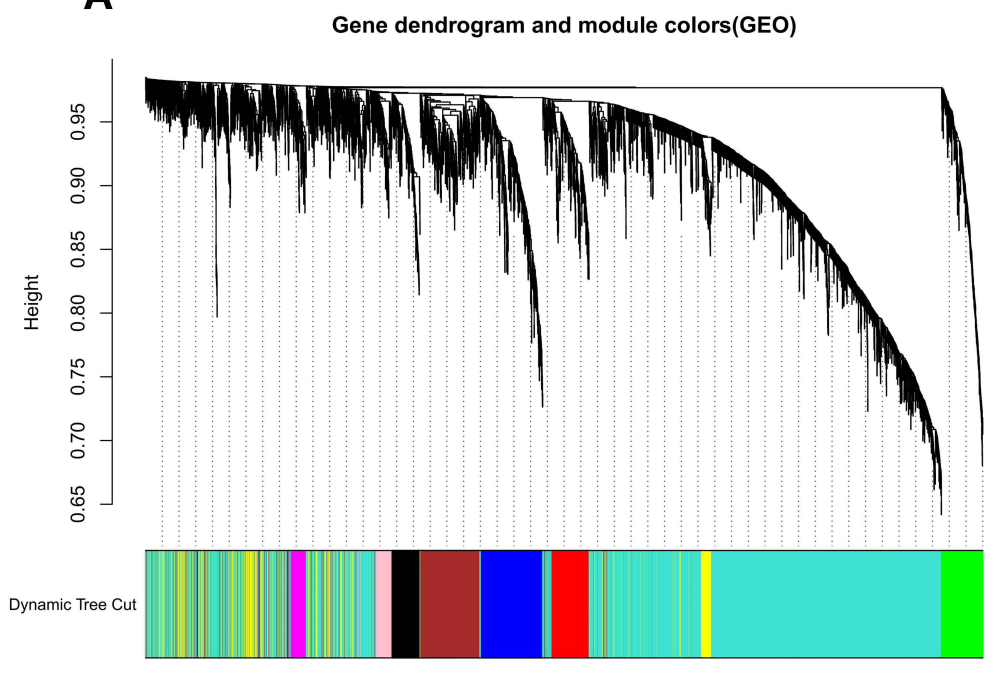

B

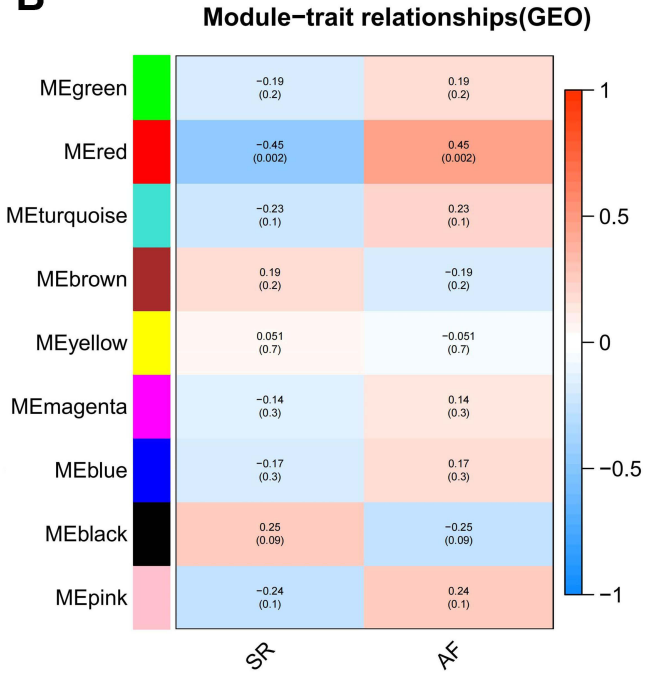

C

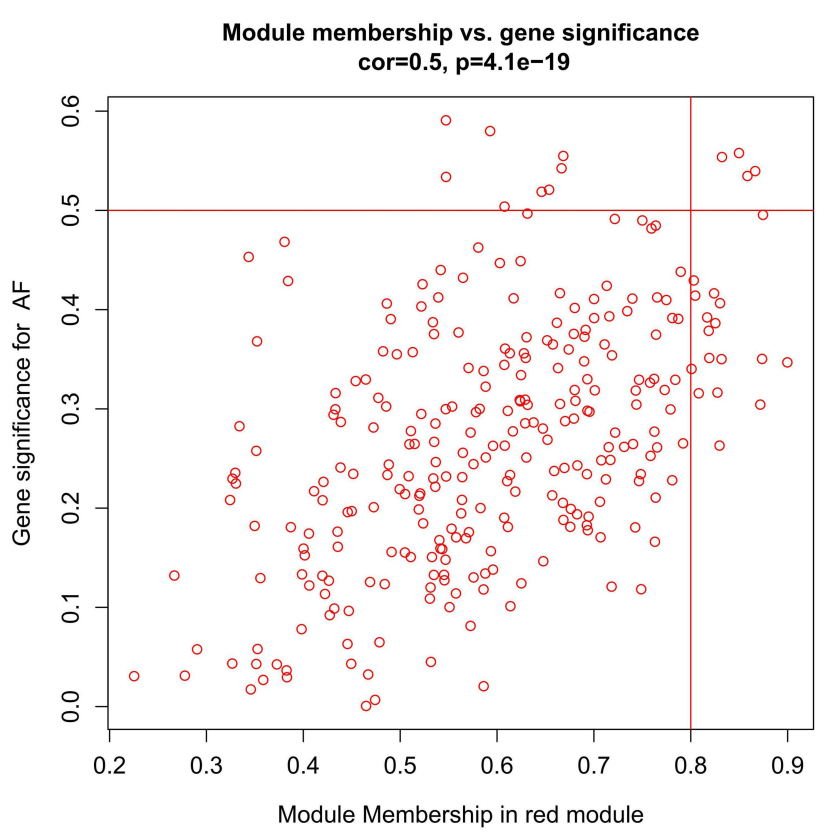

D

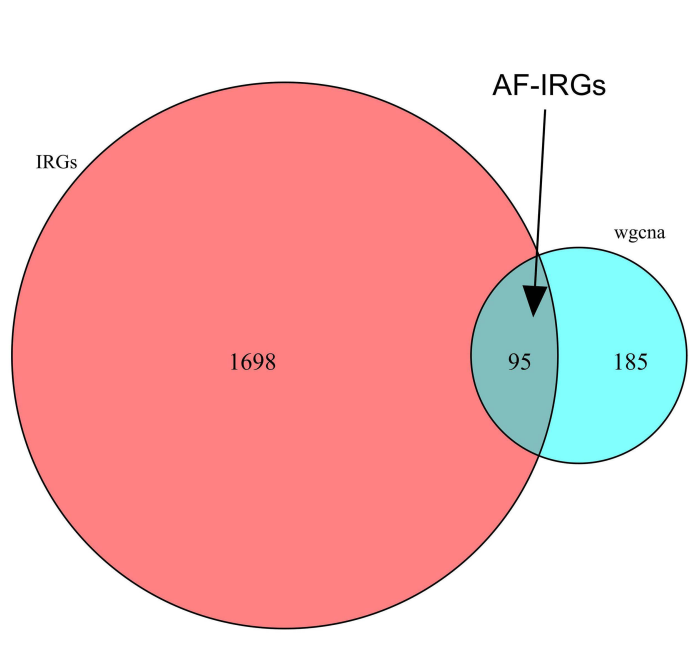

Figure 3 Identification of modules associated with AF in the integrated mRNA dataset and AF-related IRGs screening. (A) A cluster dendrogram was built based on the dissimilarity of the topological overlap. (B) Module-trait relationships. The red module was significantly correlated with AF. (C) Correlation plot between MM (X-axis) and GS (Y-axis) of genes contained in the red module. (D) Venn diagram showing intersecting genes between IRGs and the genes in the red module.

Abbreviations: AF, atrial fibrillation; IRGs, immune-related genes.

using the CSCD database. Then, 9 intersecting miRNAs were acquired by the intersection of target miRNAs and DE miRNAs (Figure 4C). Next, a total of 13,135 potential target genes were predicted for the 9 miRNAs by using the TargetScan database. Subsequently, 56 intersecting mRNAs were obtained by the intersection of target genes and AF-IRGs (Figure 4D). Finally, an immune-related circRNA-miRNA-mRNA ceRNA network in AF was constructed with 12 up-regulated circRNAs, 7 down-regulated
miRNAs and 50 up-regulated mRNAs (Figure 5A and Table 1).

\section{Enrichment Analyses for mRNAs in the Immune-Related ceRNA Network}

The results of the GO analysis showed that the identified mRNAs in the network were predominantly enriched in $\mathrm{T}$ cell activation and cell chemotaxis (biological processes, BP), external side of plasma membrane (cellular components, 
A

B

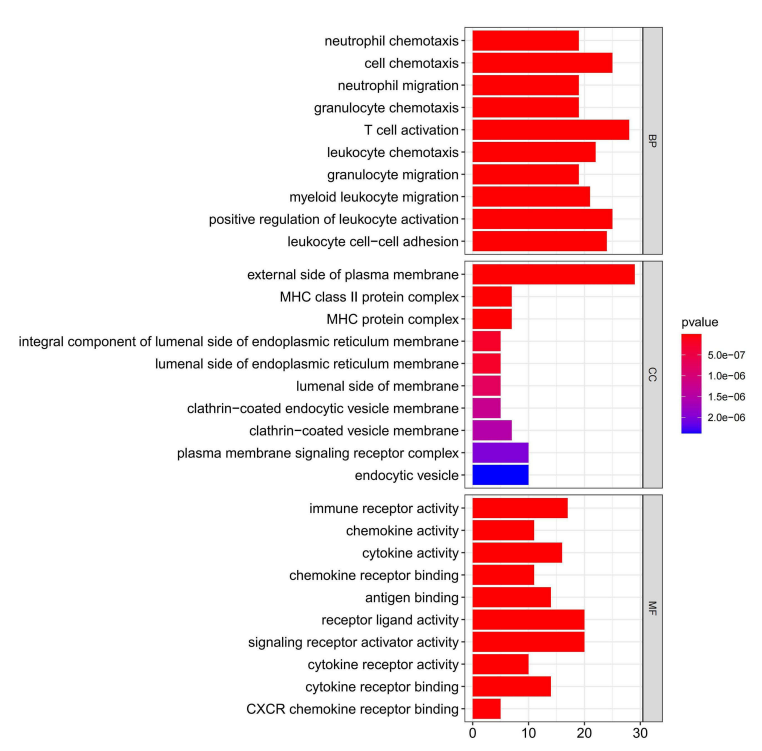

C

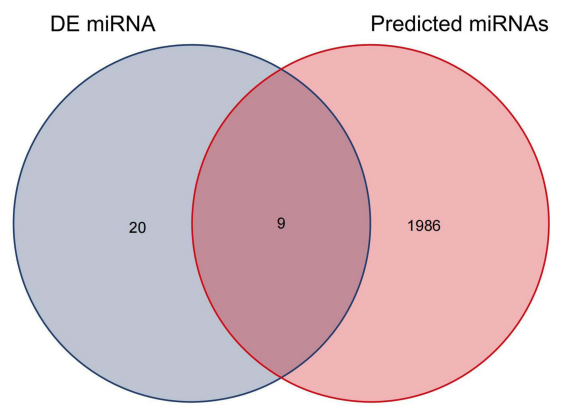

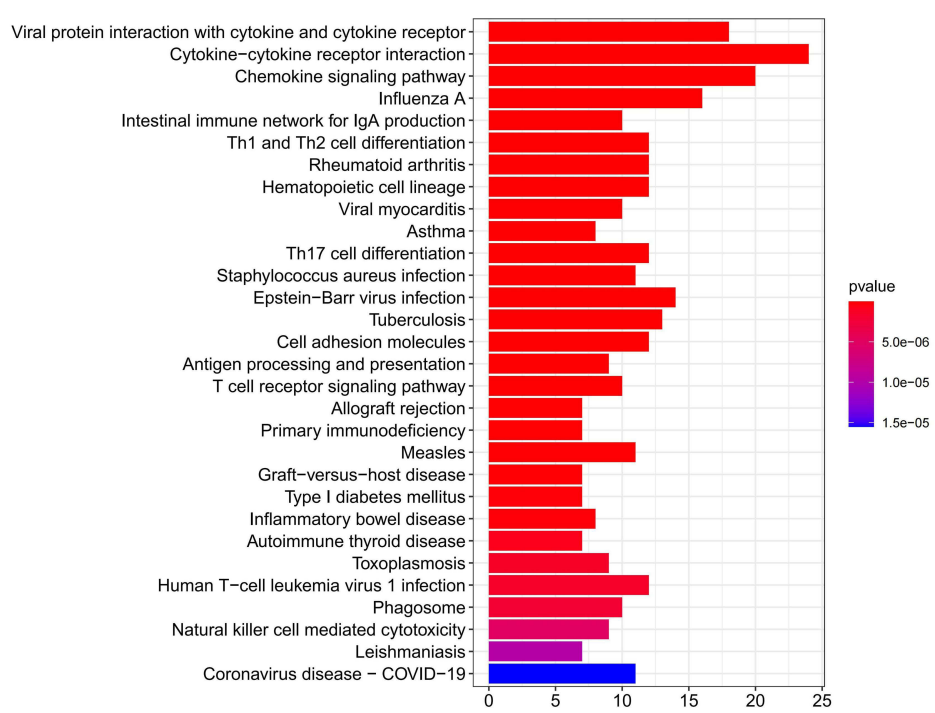

D

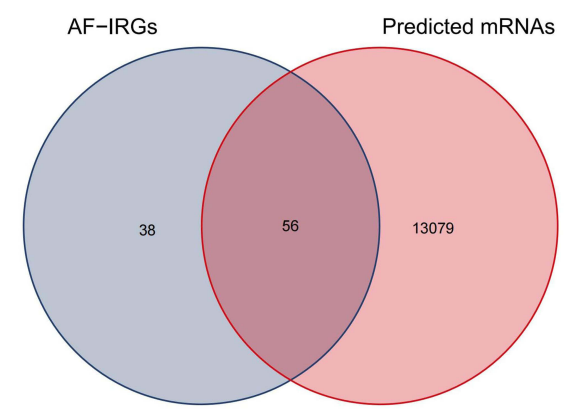

Figure 4 AF-IRGs enrichment analysis and Venn diagram. (A) GO enrichment analysis for AF-IRGs; (B) KEGG pathway enrichment analysis for AF-IRGs; (C) Venn diagram of overlapping miRNAs from predicted miRNAs and DE miRNAs in the GSE70887 dataset. (D) Venn diagram of overlapping mRNAs from AF-IRGs and predicted mRNAs. Abbreviations: AF, atrial fibrillation; AF-IRGs, AF-related immune genes; DE, differentially expressed; GO, Genome Ontology; KEGG, Kyoto Encyclopedia of Genes and Genomes.

$\mathrm{CC}$ ), and receptor ligand activity (molecular functions, MFs) (Figure 5B). KEGG pathway analysis showed significant enrichment in the cytokine and cytokine receptor interaction and chemokine signaling pathway (Figure 5C).

\section{PPI Network Analysis}

A PPI network including 47 nodes and 594 edges were built for the $50 \mathrm{mRNAs}$ of the ceRNA network by using the STRING database after removing unconnected nodes (Figure 5D). Hub genes in the PPI network were identified by using the MCC algorithm. According to the MCC sores, the top ten highest-scored genes (Figure 5E), including C-X-C motif chemokine 10 (CXCL10), C-X-C motif chemokine 9 (CXCL9), T-cell surface glycoprotein CD8 alpha chain (CD8A), Interleukin-7 (IL-7), Interleukin-7 receptor subunit alpha (IL7R), Tumor necrosis factor ligand superfamily member 13B (TNFSF13B), Stromal cell-derived factor 1 (CXCL12), CD28, C-C chemokine receptor type 5 (CCR5) and $\mathrm{C}-\mathrm{C}$ chemokine receptor type 7 (CCR7) were selected as the hub genes, and 5 of them were upregulated in the AF patients compared to the SR controls, known as DE hub genes (CD8A, IL7R, TNFSF13B, CXCL12, CCR5) (Table 2).

\section{Immune Infiltration Analyses}

The proportion of 22 immune cells detected by the CIBERSORT algorithm in each sample were displayed in the bar plot and heat map (Figure 6A and B). PCA was used to analyze the immune cells in AF and SR tissues, and it was found that there were significant group bias clustering and individual differences (Figure 6C). The correlation of 22 types of immune cells are shown in Figure 6D. Compared with SR, AF patients commonly contained a higher proportion 
B

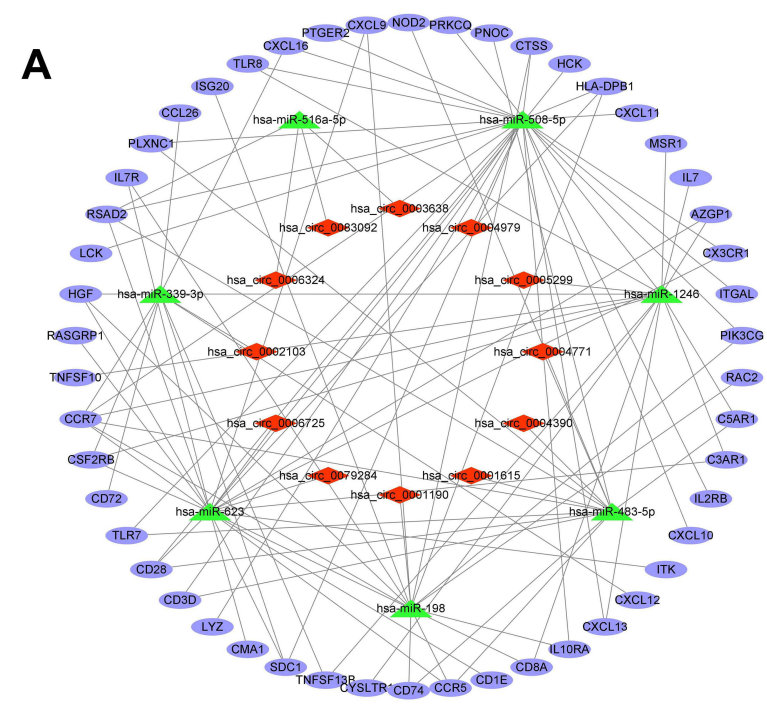

C
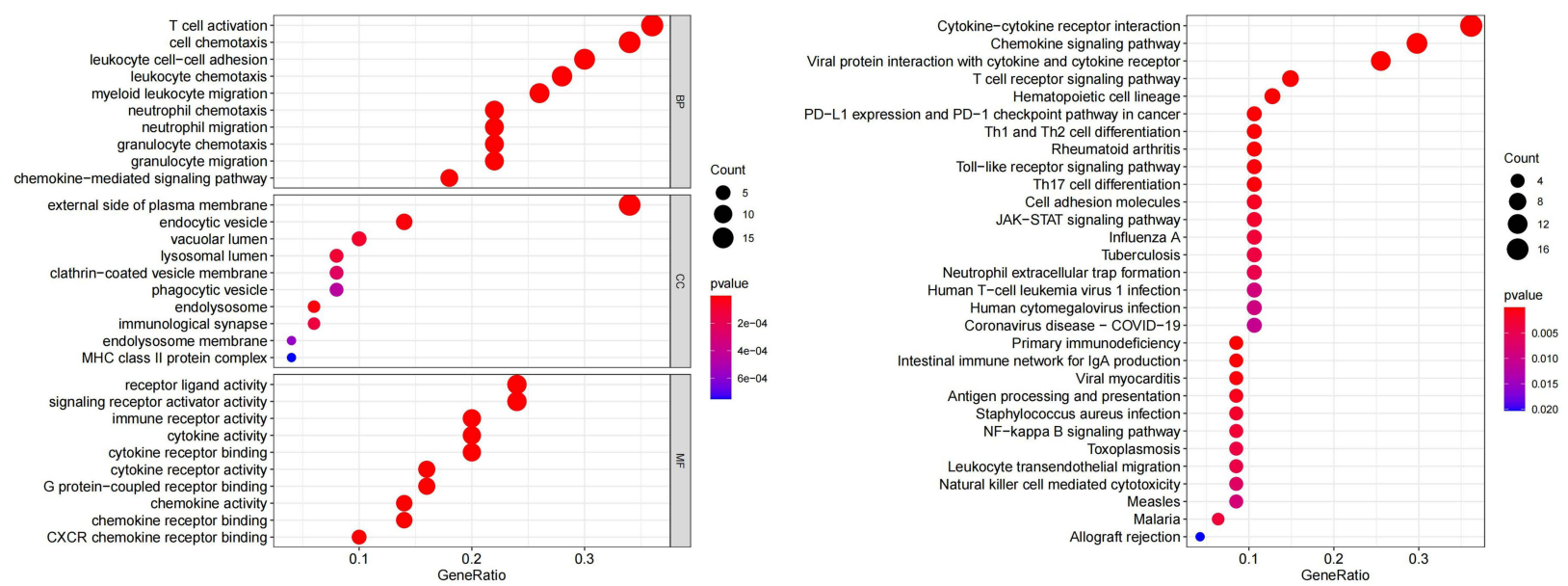

D
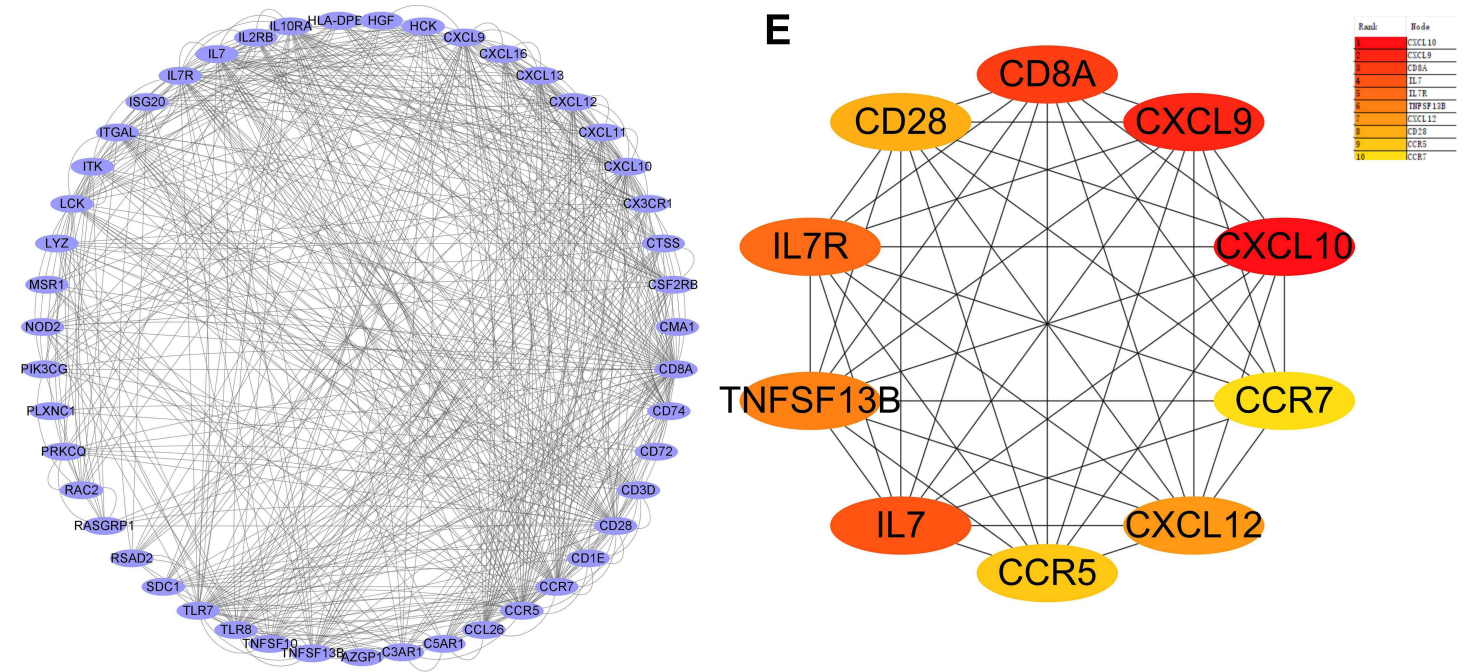

Figure 5 Construction and analysis of an immune-related ceRNA network. (A) The immune-related circRNA-miRNA-mRNA network in AF. Red diamonds indicate upregulated circRNAs, green triangles represent downregulated miRNAs, and blue ovals represent AF-IRGs; (B) GO enrichment analysis for mRNAs in the ceRNA network; (C) KEGG pathway enrichment analysis for mRNAs in the ceRNA network; (D) PPI network construction for mRNAs in the ceRNA network; (E) Hub gene selection from PPI network by using the MCC algorithm.

Abbreviations: AF, atrial fibrillation; AF-IRGs, AF-related immune genes; ceRNA, competing endogenous RNA; GO, Genome Ontology; KEGG, Kyoto Encyclopedia of Genes and Genomes; PPI, protein-protein interaction. 
Table I Immune-Related ceRNA Network of circRNAs, miRNAs, and mRNAs in AF

\begin{tabular}{|c|c|c|}
\hline circRNA & miRNA & mRNA \\
\hline hsa_circ_0005299 & $\begin{array}{l}\text { hsa-miR } \\
-1246\end{array}$ & $\begin{array}{l}\text { MSRI, CYSLTRI, TNFSFI0, HGF, } \\
\text { C5ARI, IL7, CD8A, TNFSFI3B, } \\
\text { C3ARI, CCR7, CXCLI3, PRKCQ, } \\
\text { TLR8, AZGPI, CSF2RB }\end{array}$ \\
\hline $\begin{array}{l}\text { hsa_circ_000477I } \\
\text { hsa_circ_0004390 } \\
\text { hsa_circ_00016I5 }\end{array}$ & $\begin{array}{l}\text { hsa-miR } \\
-483-5 p\end{array}$ & $\begin{array}{l}\text { C5ARI, CCR5, PLXNCI, RSAD2, } \\
\text { CCR7, CD74, CD28, TLR7, NOD2, } \\
\text { PNOC, CD3D }\end{array}$ \\
\hline hsa_circ_000 II 90 & $\begin{array}{l}\text { hsa-miR } \\
-198\end{array}$ & $\begin{array}{l}\text { ILIORA, CCR7, ISG20, CXCL9, } \\
\text { CTSS, HLA-DPBI, PIK3CG, CD74, } \\
\text { CCR5, IL7R, HGF, RAC2 }\end{array}$ \\
\hline $\begin{array}{l}\text { hsa_circ_0079284 } \\
\text { hsa_circ_0006725 }\end{array}$ & $\begin{array}{l}\text { hsa-miR } \\
-623\end{array}$ & $\begin{array}{l}\text { CMAI, CCR7, RSAD2, CXCL9, } \\
\text { HLA-DPBI, CSF2RB, C3ARI, ITK, } \\
\text { CDIE, SDCI, CCR5, RASGRPI, } \\
\text { CD28, CX3CRI, IL7R, HGF, HCK, } \\
\text { AZGPI, CD8A }\end{array}$ \\
\hline hsa_circ_0002 I03 & $\begin{array}{l}\text { hsa-miR } \\
-339-3 p\end{array}$ & $\begin{array}{l}\text { CD72, CSF2RB, CXCLI6, CXCLI2, } \\
\text { CCR7, TNFSFI3B, CCL26, SDCI }\end{array}$ \\
\hline hsa_circ_0004979 & $\begin{array}{l}\text { hsa-miR } \\
-508-5 p\end{array}$ & $\begin{array}{l}\text { RSAD2, CXCLI6, PLXNCI, } \\
\text { CX3CRI, CXCLI0, CXCLI3, LYZ, } \\
\text { SDCI, CD3D, PIK3CG, CD28, } \\
\text { TLR7, CTSS, HLA-DPBI, LCK, } \\
\text { ILIORA, TLR8, PTGER2, CCR7, } \\
\text { CXCLII, ITGAL, IL2RB }\end{array}$ \\
\hline $\begin{array}{l}\text { hsa_circ_0006324 } \\
\text { hsa_circ_0083092 } \\
\text { hsa_circ_0003638 }\end{array}$ & $\begin{array}{l}\text { hsa-miR } \\
-516 a-5 p\end{array}$ & RSAD2 \\
\hline
\end{tabular}

of neutrophils, and a lower proportion of $\mathrm{T}$ cells regulatory (Tregs) and NK cells activated $(\mathrm{P}<0.05$ ) (Figure 6E).

\section{Correlation Analysis Between DE Hub Genes and AF-Related Infiltrating Immune Cells}

A total of $4 \mathrm{DE}$ hub genes were linearly associated with Tregs or NK cells activated. Tregs were negatively associated with
CXCL12 $(R=-0.4, P=0.007), \operatorname{IL} 7 R(R=-0.36, P=0.013)$ and TNFSF13B $(\mathrm{R}=-0.38, \mathrm{P}=0.008)$. NK cells activated were negatively correlated with CD8A $(\mathrm{R}=-0.34, \mathrm{P}=$ 0.022), IL7R $(R=-0.36, P=0.013)$ and TNFSF13B $(R=$ $-0.41, \mathrm{P}=0.004$ ) (Figure 7).

\section{Construction of the Immune Cells-Related ceRNA Subnetwork and ROC Curve Analysis}

Four DE hub genes (CD8A, IL7R, TNFSF13B, and CXCL12) associated with AF-related immune cells (Tregs and NK cells activated), 4 miRNAs and 6 circRNAs were eventually used to construct immune cellsrelated ceRNA subnetwork (Figure 8). The expression levels of cicrRNAs, miRNAs and mRNAs in the ceRNA subnetwork in their respective datasets are shown in Figure 9. The expression of two genes (TNFSF13B and CXCL12) were significantly associated with a diagnosis of $\mathrm{AF}(0.7<\mathrm{AUC}<1)$ by ROC curve analysis (Figure 10$)$.

\section{Discussion}

In recent years, more and more evidences have revealed that the pathogenesis of AF is related to immune and inflammatory responses. And emerging data have elucidated that circRNA-miRNA-mRNA ceRNA network is implicated in the pathological progression of cardiovascular disease. Therefore, we sought to perform a combined analysis of a ceRNA immunoregulatory network and tissue-infiltrating immune cells in AF to provide theoretical basis for finding new targets for the prevention and treatment of AF.

Firstly, we screened 95 AF-IRGs, and the enrichment function confirmed that these genes were primarily associated with immune and inflammatory responses. Together with AF-IRGs, we constructed an immune-related circRNA-miRNA-mRNA ceRNA network in AF, indicating that these circRNAs, miRNAs and mRNAs in the

Table 2 DE Hub Genes with Higher MCC Score in Protein-Protein Interaction Network

\begin{tabular}{|l|c|c|c|l|}
\hline Gene Symbol & MCC Score & logFC & P value & Gene Title \\
\hline CD8A & 473738 & 0.431 & 0.036 & T-cell surface glycoprotein CD8 alpha chain \\
IL7R & 342360 & 0.440 & 0.031 & Interleukin-7 receptor subunit alpha \\
TNFSFI3B & 339480 & 0.487 & 0.018 & Tumor necrosis factor ligand superfamily member I3B \\
CXCLI2 & 285,522 & 0.500 & 0.012 & Stromal cell-derived factor I \\
CCR5 & 238,786 & 0.504 & 0.005 & C-C chemokine receptor type 5 \\
\hline
\end{tabular}

Note: Positive logFC values correspond to up-regulated genes.

Abbreviations: DE, differentially expressed; FC, fold change; MCC, maximal clique centrality. 

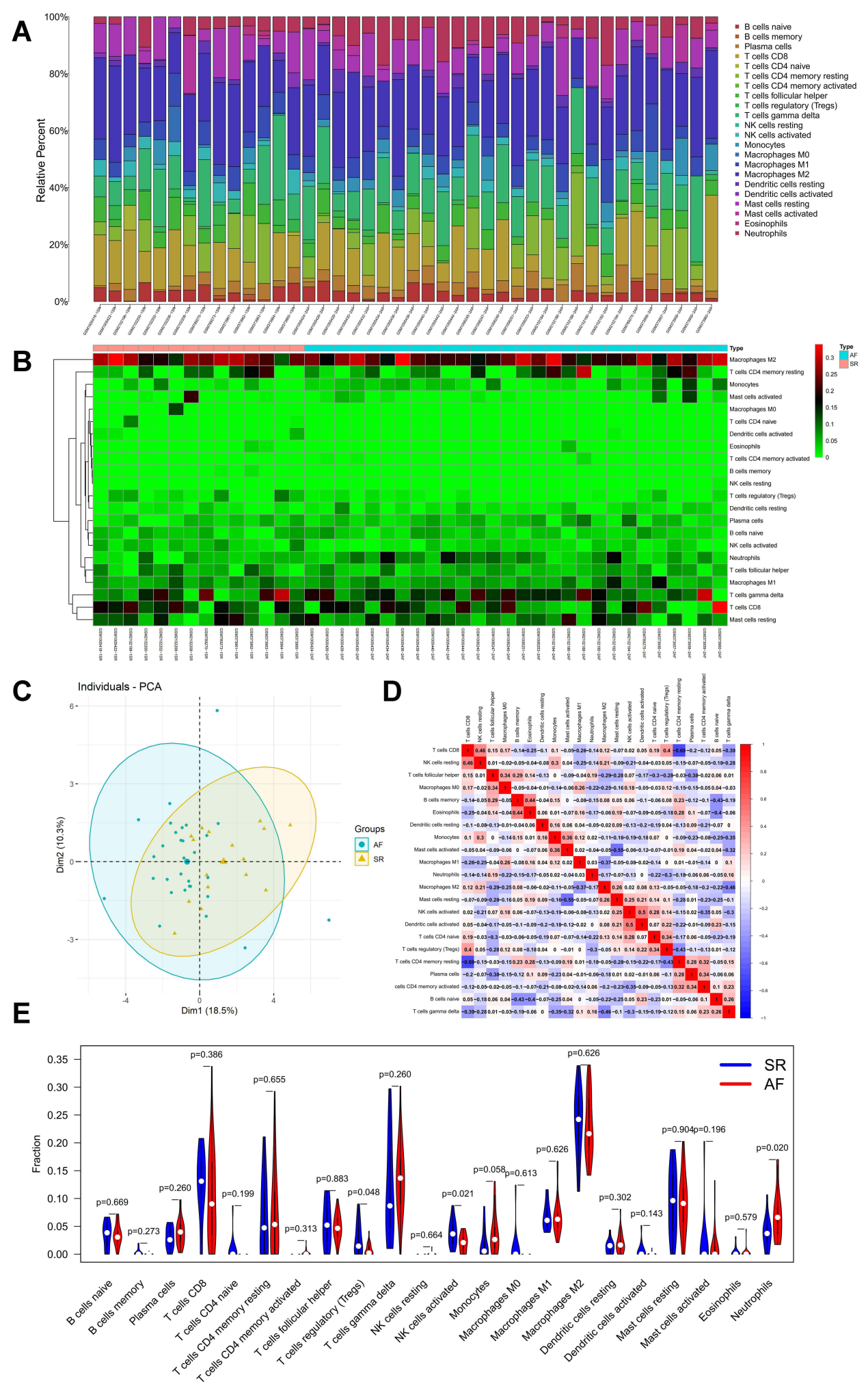

Figure 6 Immune infiltration analyses for AF. The proportion (A) and heatmap (B) of 22 immune cells detected by CIBERSORT algorithm. (C) The principal component analysis for immune cell in AF and SR tissues. (D) Correlation heat map for immune cells. (E) The violin plot of immune cells.

Abbreviations: $A F$, atrial fibrillation; $S R$, sinus rhythm.

network may play significant roles in AF immune mechanisms. The results of the GO enrichment analysis showed that 50 mRNAs in the ceRNA immunoregulatory network were also mainly implicated in the inflammation and immune system. Further, KEGG pathway analysis results were predominantly enriched in cytokine-cytokine 
A
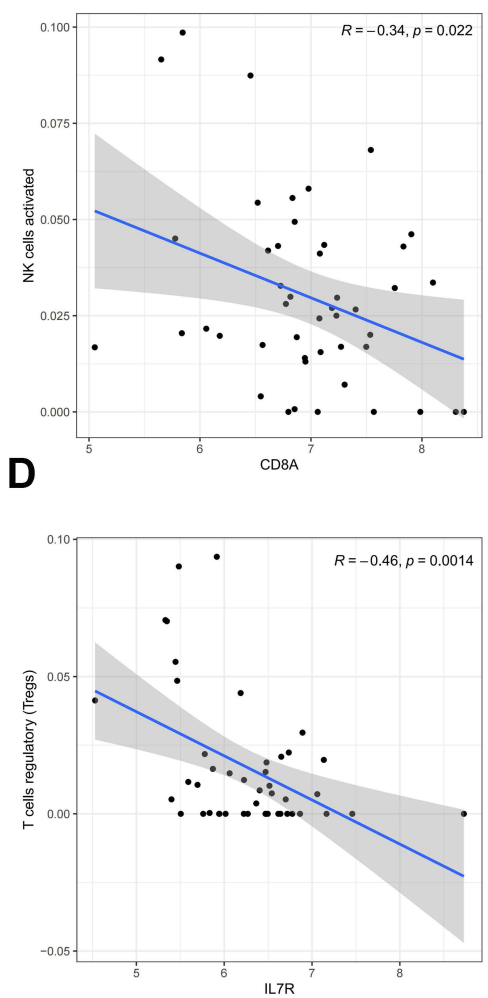

B
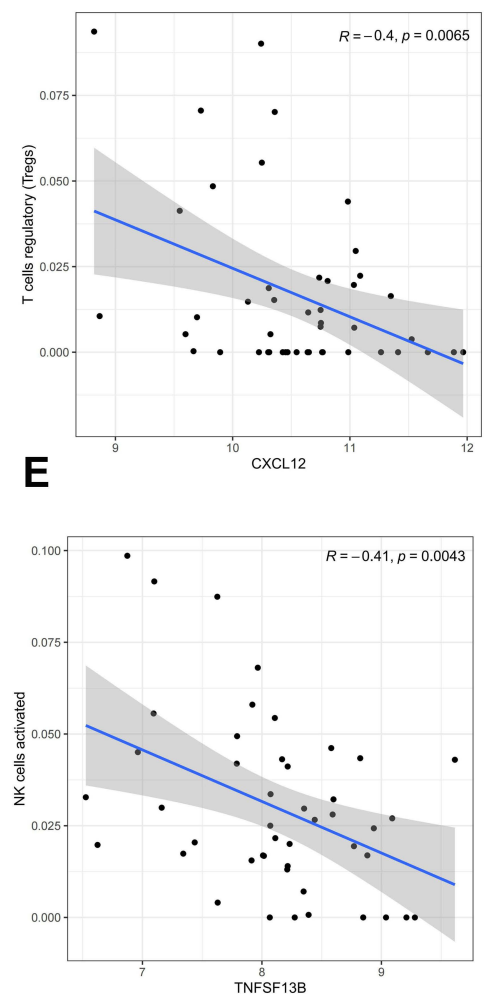

C
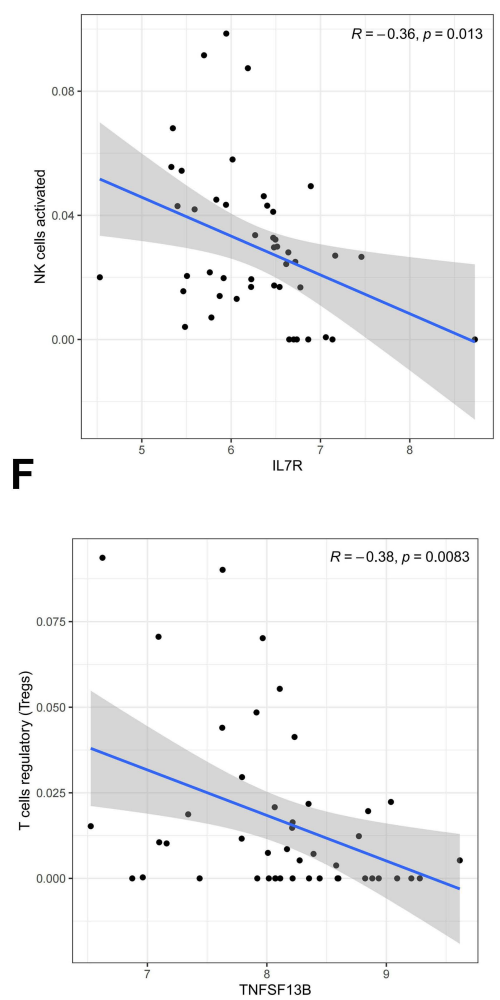

Figure 7 The correlation between DE hub genes and AF-related immune cells. The relationships between DE hub genes and AF-related immune cells were calculated using Pearson correlation coefficients (A-F).

Abbreviations: $A F$, atrial fibrillation; $D E$, differentially expressed.

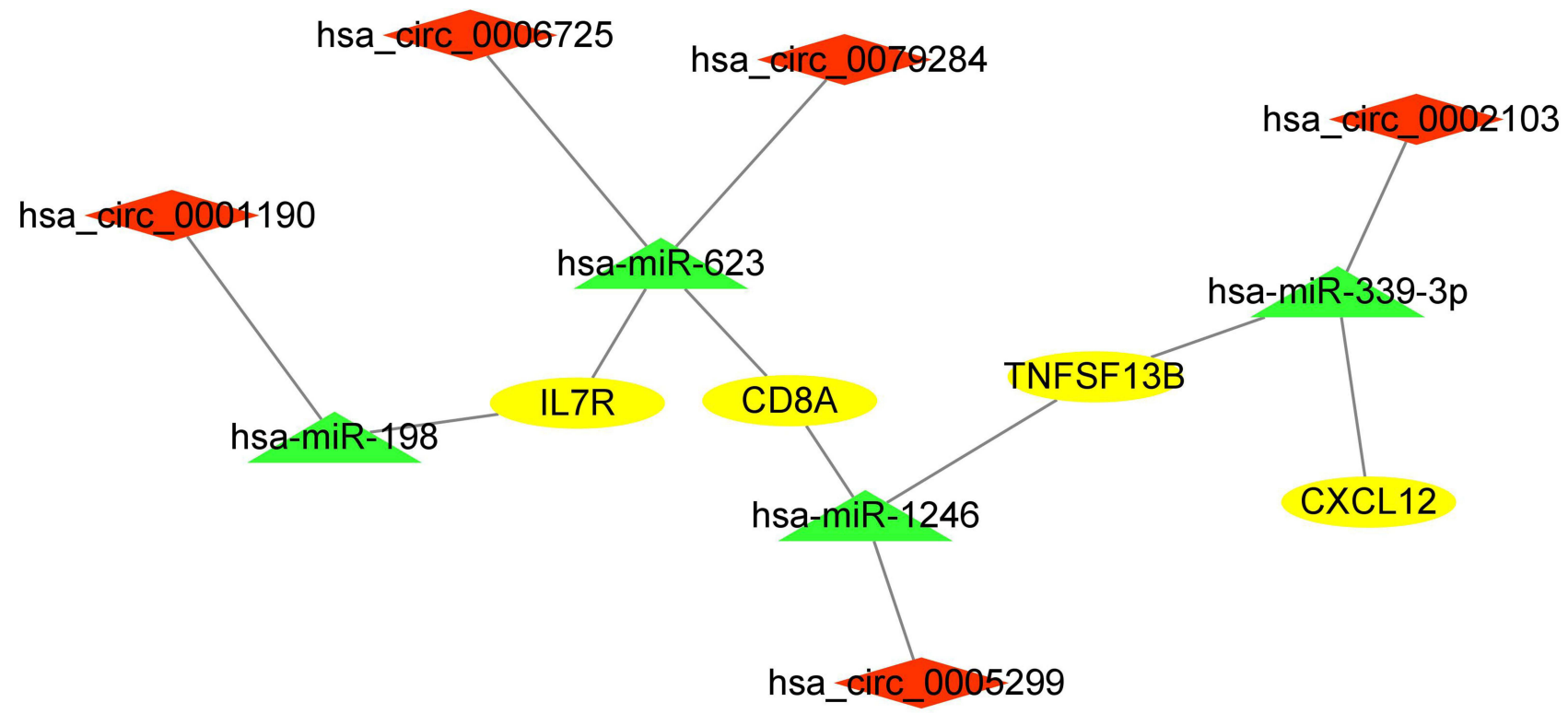

Figure 8 Construction of the immune cells-related circRNA-miRNA-mRNA subnetwork. Red diamonds indicate upregulated circRNAs, green triangles represent downregulated miRNAs, and yellow ovals represent upregulated mRNAs.

receptor interactions and the chemokine signaling pathway, which showed a close relationship with myocardial ischemia/reperfusion injury as reported in Liu et al's study. ${ }^{31}$ Some studies have shown that chemokines and their receptors regulate immune cell recruitment and activation and participate in persisting of the local 


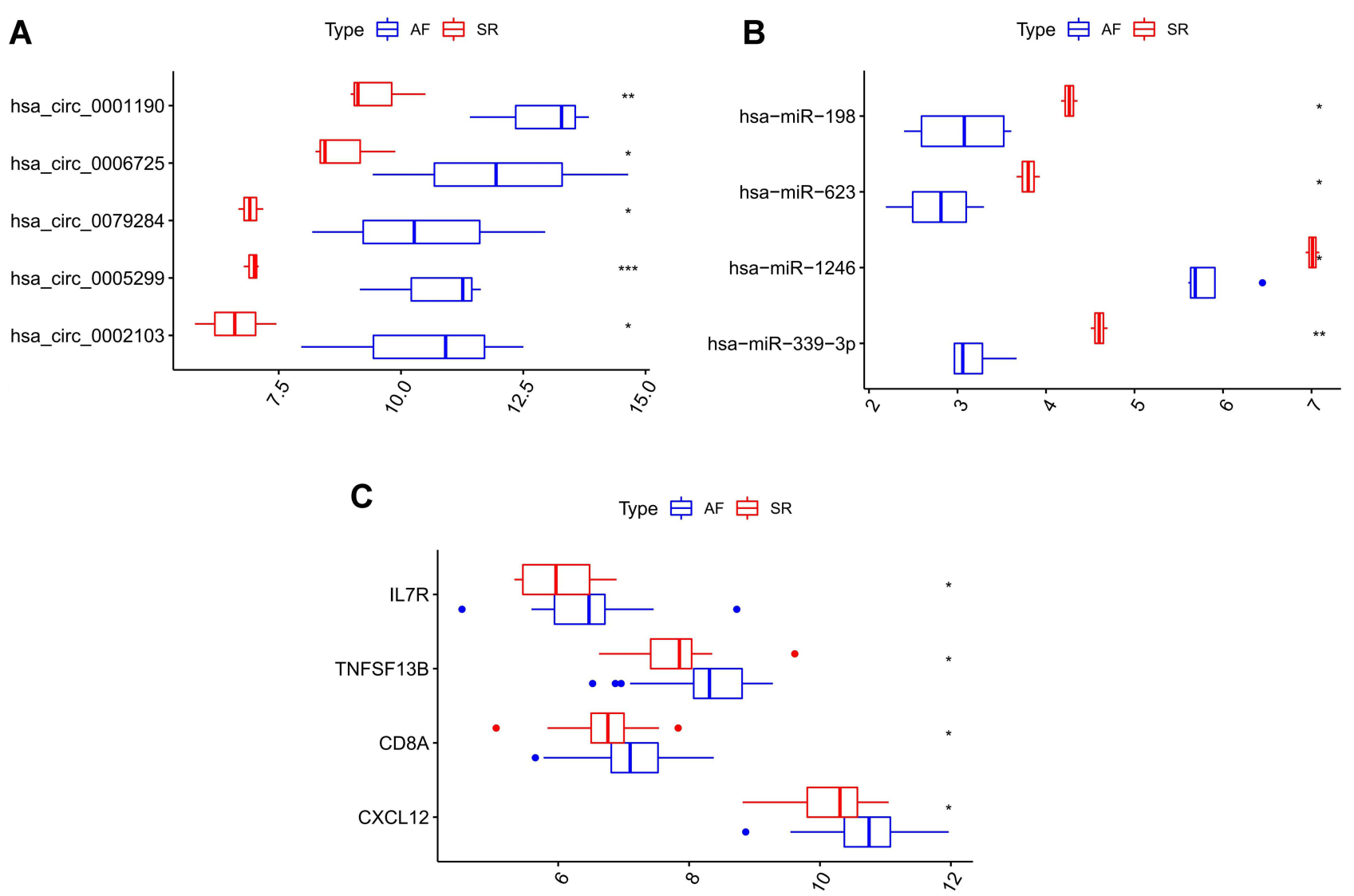

Figure 9 The boxplot maps of cicrRNAs (A) miRNAs (B) and mRNAs (C) expression levels in the immune cells-related ceRNA subnetwork in their respective datasets. $* \mathrm{P}<0.05$, ** $\mathrm{P}<0.01$, *** $\mathrm{P}<0.001$.

inflammatory response in atherosclerosis, ${ }^{32}$ as well as the pathophysiology of left ventricular remodeling and cardiac dysfunction caused by pressure overload. ${ }^{33}$ These findings indicated that cytokine-cytokine receptor interactions and the chemokine signaling pathway play an important role in the cardiovascular field, which may be associated with the development of AF. Subsequently, a PPI network was established by using mRNAs in the ceRNA immunoregulatory network and we filtered $5 \mathrm{DE}$ hub genes (CD8A, IL7R, TNFSF13B, CXCL12, CCR5). Among them, 4 hub genes (CCR5, CXCL12, TNFSF13B, IL7R) were enriched in cytokine-cytokine receptor interactions and 2 hub genes (CCR5, CXCL12) were enriched in chemokine signaling pathway. Therefore, our results suggested that these DE hub genes may be primarily involved in the immune and inflammatory processes of AF.

Next, immune infiltration was investigated in AF by CIBERSORT. In this program, we found that a higher proportion of neutrophils, as well as a lower proportion of $\mathrm{T}$ cells regulatory (Tregs) and NK cells activated may be associated with the pathogenesis of AF. Neutrophils are the most abundant circulating leukocyte in humans, and study found that myocardial inflammatory infiltration may be a cause of AF, including neutrophil and inflammation markers. ${ }^{34}$ Moreover, $\mathrm{Wu}$ et al ${ }^{35}$ showed that neutrophil-to -lymphocyte ratio could be an independent predictor of long-term outcomes in patients with AF. Chen et $\mathrm{al}^{36}$ showed that the proportion of Tregs was obviously lower in AF patients, which was consistent with the results of our study. Another study confirmed that Tregs mitigate cardiac hypertrophy and myocardial fibrosis in Angiotensin II-induced hypertensive mice. ${ }^{37} \mathrm{NK}$ cells account for more than $1 \%$ of cardiac lymphocytes and are involved in the regulation of cardiac diseases. ${ }^{38}$ Reduction in the number and function of NK cells has been found in patients with coronary artery disease $(\mathrm{CAD}),{ }^{39}$ especially in patients with acute coronary syndrome. ${ }^{40}$ In addition, IFN- $\gamma$-producing NK cells have been demonstrated to negatively regulate liver fibrosis, suggesting that low expression of NK cells may be involved in atrial fibrosis, ${ }^{41}$ which requires further experimental verification. Therefore, neutrophils, Tregs and NK 


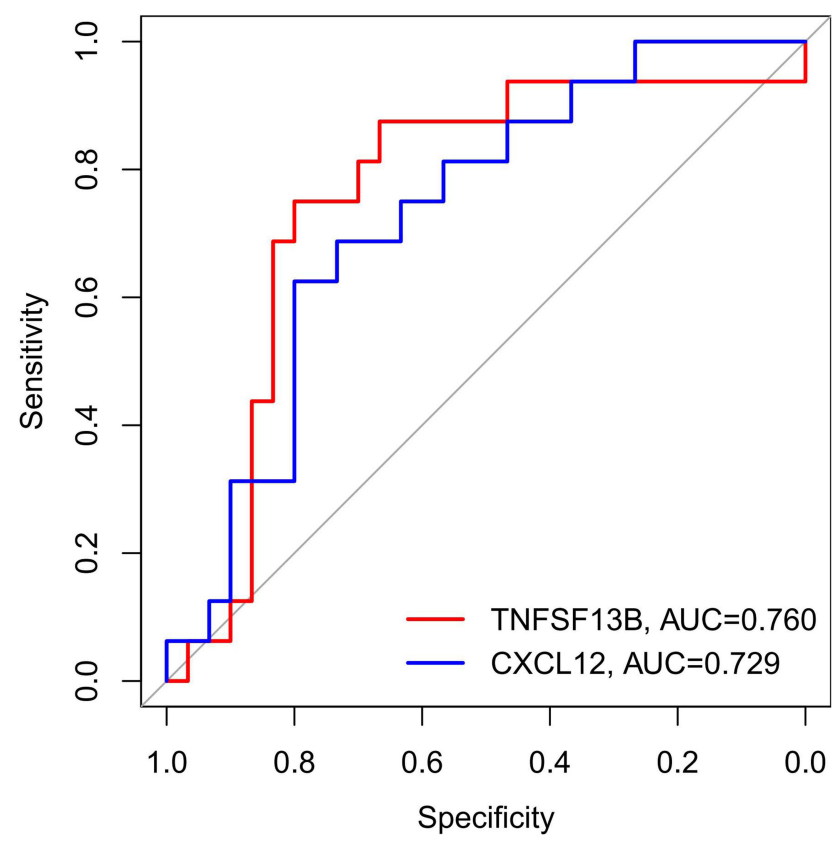

Figure 10 The receiver operator characteristic curves of CXCLI 2 and TNFSFI3B for AF.

Abbreviation: $A F$, atrial fibrillation.

cells may be potential key subtypes implicated in driving the progression of AF disease.

Ulteriorly, our results found that only $4 \mathrm{DE}$ hub genes (CD8A, IL7R, TNFSF13B, and CXCL12) were associated with Tregs or NK cells activated, but not with neutrophils by Pearson correlation coefficients, and we constructed the immune cells-related ceRNA subnetwork. And only CXCL12 and TNFSF13B could accurately predict AF $(0.7<$ AUC $<1)$ by ROC curve analysis, indicating that the two genes play a key role in the pathogenesis of AF. Study has been reported that plasma stromal cell -derived factor 1 (SDF-1, also called CXCL12) was increased in patients with AF compared with patients with $\mathrm{SR}^{42}$ $\mathrm{C}-\mathrm{X}-\mathrm{C}$ chemokine receptor type 4 (CXCR4) is an $\alpha$-chemokine receptor specific for CXCL12 that transduces signals by increasing intracellular calcium ion levels and enhancing MAPK1/MAPK3 activation, ${ }^{43,44}$ and might contribute to the process of AF through regulating atrial fibrosis and structural remodeling. ${ }^{45}$ Recently, one study ${ }^{46}$ has shown that the CXCL12/CXCR4 axis as a key mediator in $\mathrm{AF}$ is involved in the progress of inflammation and fibrosis. Therefore, the CXCL12/CXCR4 axis plays an important role in the pathogenesis of AF. The immune cells-related ceRNA subnetwork showed that downregulated miR-339-3p and target upregulated CXCL12 were under the regulation of has_circ_0002103. Wu et $\mathrm{al}^{47}$ revealed that overexpressed miR-339-3p could suppress Anxa3 to inhibit the Akt/mTOR signaling pathway, so as to alleviate tissue edema, inflammation in mice with severe acute pancreatitis-associated acute lung injury. And the mTOR signaling pathway plays an important role in the heart against stress and cardiomyocyte survival, ${ }^{48,49}$ which may be related to the development of AF. So, downexpressed miR-339-3p may be involved in the immune and inflammatory processes in AF. In parallel, Nemo-like kinase (NLK) is the host gene of has_circRNA_0002103, and regulates the Wnt signaling pathway, whose dysregulation can lead to immunodeficiency, chronic inflammation, and cancer. ${ }^{50}$ And study has showed that an inducible transgenic mouse with cardiac-specific NLK expression was more susceptible to left ventricular damage and heart failure. ${ }^{51}$ So, has_circRNA_0002103 may regulate the immune inflammatory mechanism of AF. These reports suggested that the interaction has_circRNA_0002103-miR-339-3p-CXCL12 regulatory axis associated with Tregs may be related to the immune mechanism of AF.

Human B cell activating factor (TNFSF13B, BAFF) is a tumor necrosis factor superfamily member. Study has shown that BAFF can inhibit Tregs, thereby promoting allergic inflammation of the airway, ${ }^{52}$ and another study has also shown that BAFF is involved in the process of myocardial fibrosis after acute myocardial infarction (MI) ${ }^{53}$ Our results found that Tregs expression was low in the left atrial tissue of AF patients and was negatively correlated with TNFSF13B. These reports suggested TNFSF13B may be involved in the immune inflammatory mechanism of AF. Additionally, the sub-network showed that downregulated miR-1246 and miR-339-3p, and target upregulated TNFSF13B were under the regulation of has_circ_0005299 and has_circ_0002103. Huang et $\mathrm{al}^{54}$ revealed that miR-1246 in exosomes derived from Endothelial progenitor cells can enhance angiogenesis in vitro and in vivo after MI, and these improvements may be related to amelioration of myocardial injury and myocardial fibrosis after MI. So, we speculated that the low expression of miR-1246 in the heart tissue of AF patients may be related to promoting cardiac fibrosis. Therefore, we speculated that has_circ_0005299-miR -1236-TNFSF13B axis and has_circ_0002103-miR-3393p-TNFSF13B axis associated with Tregs and NK cells might be related to the immune mechanism of AF.

The remaining two genes (CD8A, IL7R) in the immune-cells-related ceRNA subnetwork have not been 
reported in AF studies, but animal study ${ }^{55}$ has suggested that $\mathrm{CD} 8 \mathrm{~A}+$ immune cell infiltration may be related to cardiac fibrosis and left ventricular contractility decline in diabetic cardiomyopathy and another study ${ }^{56}$ has shown that IL7R is involved in the inflammatory response in atherosclerosis. As for the immune cells-related ceRNA subnetwork constructed by us, it was found that the upregulated circRNAs (has_circ_0001190, has_circ_0006725, has_circ_0079284, and has_circ_0005299), downregulated miRNAs (has-miR-198, has-miR-623, and has-miR-1246) and upregulated mRNAs (CD8A and IL7R) may be immunologically related to $\mathrm{AF}$, but the specific mechanism still needs further study.

However, our study has some limitations. First, AF sample size for circRNA and miRNA microarray analysis was small in the present study. Second, the samples analyzed for miRNA were not from the left atrial appendage, and the difference between the left and right atrial in AF patients may bias the results. Thirdly, for better clinical significance, the biomarkers for the diagnosis of AF should be verified in blood samples of AF patients. Finally, the results of this study need to be further verified by future in vivo or in vitro experiments.

\section{Conclusion}

Our study found that an immune-related circRNA-miRNA -mRNA ceRNA network in AF which includes 5 circRNAs (has_circ_0001190, has_circ_0006725, has_circ_0079284, has_circ_0005299, and has_circ_0002103), 4 miRNAs (has-miR-198, has-miR-623, has-miR-1246, and has-miR-339-3p) and 4 mRNAs (CXCL12, IL7R, TNFSF13B, and CD8A) may depend on the interaction among tissue-infiltrating immune cells, including Tregs or NK cells activated. Our results may provide new insights into the molecular mechanisms governing AF progression, and provide theoretical basis for finding new targets for the prevention and treatment of AF.

\section{Abbreviations}

AF, atrial fibrillation; ceRNA, competing endogenous RNA; CCR5, C-C chemokine receptor type 5; CCR7, C-C chemokine receptor type 7; CXCL9, C-X-C motif chemokine 9; CXCL10, C-X-C motif chemokine 10; CXCL12, Stromal cell-derived factor 1; GO, Genome Ontology; IL-7, Interleukin-7; IL7R, Interleukin-7 receptor subunit alpha; IRGs, immune-related genes; KEGG, Kyoto Encyclopedia of Genes and Genomes; PPI, protein-protein interaction;
ROC, receiver operating characteristic; TNFSF13B, Tumor necrosis factor ligand superfamily member.

\section{Data Sharing Statement}

The data in this study are available from the corresponding author (Mingxing $\mathrm{Wu}$ ) on a reasonable request.

\section{Author Contributions}

All authors contributed to data analysis, drafting or revising the article, have agreed on the journal to which the article will be submitted, gave final approval for the version to be published, and agree to be accountable for all aspects of the work.

\section{Disclosure}

The authors report no conflicts of interest in this work.

\section{References}

1. Staerk L, Sherer JA, Ko D, Benjamin EJ, Helm RH. Atrial fibrillation: epidemiology, pathophysiology, and clinical outcomes. Circ Res. 2017;120(9):1501-1517. doi:10.1161/CIRCRESAHA.117.309 732

2. Nattel S. Molecular and cellular mechanisms of atrial fibrosis in atrial fibrillation. JACC Clin Electrophysiol. 2017;3(5):425-435. doi:10. 1016/j.jacep.2017.03.002

3. Hu YF, Chen YJ, Lin YJ, Chen SA. Inflammation and the pathogenesis of atrial fibrillation. Nat Rev Cardiol. 2015;12(4):230-243. doi:10.1038/nrcardio.2015.2

4. Liu Y, Shi Q, Ma Y, Liu Q. The role of immune cells in atrial fibrillation. J Mol Cell Cardiol. 2018;123:198-208. doi:10.1016/j. yjmcc.2018.09.007

5. Conway DS, Buggins P, Hughes E, Lip GY. Relationship of interleukin-6 and C-reactive protein to the prothrombotic state in chronic atrial fibrillation. $J$ Am Coll Cardiol. 2004;43(11): 2075-2082. doi:10.1016/j.jacc.2003.11.062

6. Wang L, Zhang YL, Lin QY, et al. CXCL1-CXCR2 axis mediates angiotensin II-induced cardiac hypertrophy and remodelling through regulation of monocyte infiltration. Eur Heart J. 2018;39 (20):1818-1831. doi:10.1093/eurheartj/ehy085

7. Cambien B, Karimdjee BF, Richard-Fiardo P, et al. Organ-specific inhibition of metastatic colon carcinoma by CXCR3 antagonism. $\mathrm{Br}$ J Cancer. 2009;100(11):1755-1764. doi:10.1038/sj.bjc.6605078

8. Miyosawa K, Iwata H, Minami-Takano A, et al. Enhanced monocyte migratory activity in the pathogenesis of structural remodeling in atrial fibrillation. PLoS One. 2020;15(10):e0240540. doi:10.1371/ journal.pone. 0240540

9. Salmena L, Poliseno L, Tay Y, Kats L, Pandolfi PP. A ceRNA hypothesis: the Rosetta stone of a hidden RNA language? Cell. 2011;146(3):353-358. doi:10.1016/j.cell.2011.07.014

10. Ashwal-Fluss R, Meyer M, Pamudurti NR, et al. circRNA biogenesis competes with pre-mRNA splicing. Mol Cell. 2014;56(1):55-66. doi:10.1016/j.molcel.2014.08.019

11. Li Z, Huang C, Bao C, et al. Exon-intron circular RNAs regulate transcription in the nucleus. Nat Struct Mol Biol. 2015;22 (3):256-264. doi:10.1038/nsmb.2959

12. Geng HH, Li R, Su YM, et al. The circular RNA Cdrlas promotes myocardial infarction by mediating the regulation of miR-7a on its target genes expression. PLoS One. 2016;11(3):e0151753. doi:10. 1371/journal.pone. 0151753 
13. Tang CM, Zhang M, Huang L, et al. CircRNA 000203 enhances the expression of fibrosis-associated genes by derepressing targets of miR-26b-5p, Colla2 and CTGF, in cardiac fibroblasts. Sci Rep. 2017;7(1):40342. doi:10.1038/srep40342

14. Newman AM, Liu CL, Green MR, et al. Robust enumeration of cell subsets from tissue expression profiles. Nat Methods. 2015;12 (5):453-457. doi:10.1038/nmeth.3337

15. Jiang S, Guo C, Zhang W, et al. The integrative regulatory network of circRNA, microRNA, and mRNA in atrial fibrillation. Front Genet. 2019;10:526. doi:10.3389/fgene.2019.00526

16. Cañón S, Caballero R, Herraiz-Martínez A, et al. miR-208b upregulation interferes with calcium handling in HL-1 atrial myocytes: implications in human chronic atrial fibrillation. J Mol Cell Cardiol. 2016;99:162-173. doi:10.1016/j.yjmcc.2016.08.012

17. Yeh YH, Kuo CT, Lee YS, et al. Region-specific gene expression profiles in the left atria of patients with valvular atrial fibrillation. Heart Rhythm. 2013;10(3):383-391. doi:10.1016/j.hrthm.2012.11.013

18. Tsai FC, Lin YC, Chang SH, et al. Differential left-to-right atria gene expression ratio in human sinus rhythm and atrial fibrillation: implications for arrhythmogenesis and thrombogenesis. Int J Cardiol. 2016;222:104-112. doi:10.1016/j.ijcard.2016.07.103

19. Doñate Puertas R, Meugnier E, Romestaing C, et al. Atrial fibrillation is associated with hypermethylation in human left atrium, and treatment with decitabine reduces atrial tachyarrhythmias in spontaneously hypertensive rats. Transl Res. 2017;184:57-67.e55 doi:10.1016/j.trs1.2017.03.004

20. Adam O, Lavall D, Theobald K, et al. Rac1-induced connective tissue growth factor regulates connexin 43 and $\mathrm{N}$-cadherin expression in atrial fibrillation. $J \mathrm{Am}$ Coll Cardiol. 2010;55(5):469-480. doi:10.1016/j.jacc.2009.08.064

21. Barrett T, Troup DB, Wilhite SE, et al. NCBI GEO: mining tens of millions of expression profiles-database and tools update. Nucleic Acids Res. 2007;35(Database):D760-765. doi:10.1093/nar/gk1887

22. Irizarry RA, Hobbs B, Collin F, et al. Exploration, normalization, and summaries of high density oligonucleotide array probe level data. Biostatistics. 2003;4(2):249-264. doi:10.1093/biostatistics/4.2.249

23. Leek JT, Johnson WE, Parker HS, Jaffe AE, Storey JD. The sva package for removing batch effects and other unwanted variation in high-throughput experiments. Bioinformatics. 2012;28(6):882-883. doi:10.1093/bioinformatics/bts034

24. Diboun I, Wernisch L, Orengo CA, Koltzenburg M. Microarray analysis after RNA amplification can detect pronounced differences in gene expression using limma. BMC Genomics. 2006;7(1):252. doi:10.1186/1471-2164-7-252

25. Ritchie ME, Phipson B, Wu D, et al. limma powers differential expression analyses for RNA-sequencing and microarray studies. Nucleic Acids Res. 2015;43(7):e47. doi:10.1093/nar/gkv007

26. Langfelder P, Horvath S. WGCNA: an R package for weighted correlation network analysis. BMC Bioinform. 2008;9(1):559. doi:10.1186/1471-2105-9-559

27. Glažar P, Papavasileiou P, Rajewsky N. circBase: a database for circular RNAs. RNA. 2014;20(11):1666-1670. doi:10.1261/rna.043687.113

28. Xia S, Feng J, Chen K, et al. CSCD: a database for cancer-specific circular RNAs. Nucleic Acids Res. 2018;46(D1):D925-D929. doi:10.1093/nar/gkx863

29. Lewis BP, Shih IH, Jones-Rhoades MW, Bartel DP, Burge CB. Prediction of mammalian microRNA targets. Cell. 2003;115 (7):787-798. doi:10.1016/S0092-8674(03)01018-3

30. Szklarczyk D, Morris JH, Cook H, et al. The STRING database in 2017: quality-controlled protein-protein association networks, made broadly accessible. Nucleic Acids Res. 2017;45(D1):D362-d368. doi:10.1093/nar/gkw937

31. Liu Y, Li G, Lu H, et al. Expression profiling and ontology analysis of long noncoding RNAs in post-ischemic heart and their implied roles in ischemia/reperfusion injury. Gene. 2014;543(1):15-21. doi:10.1016/j.gene.2014.04.016
32. Bakogiannis C, Sachse M, Stamatelopoulos K, Stellos K. Plateletderived chemokines in inflammation and atherosclerosis. Cytokine. 2019;122:154157. doi:10.1016/j.cyto.2017.09.013

33. Finsen AV, Ueland T, Sjaastad I, et al. The homeostatic chemokine CCL21 predicts mortality in aortic stenosis patients and modulates left ventricular remodeling. PLoS One. 2014;9(11):e112172. doi:10.1371/journal.pone.0112172

34. Fontes ML, Mathew JP, Rinder HM, Zelterman D, Smith BR, Rinder CS. Atrial fibrillation after cardiac surgery/cardiopulmonary bypass is associated with monocyte activation. Anesth Analg. 2005;101(1):17-23. doi:10.1213/01.ANE.0000155260.93406.29

35. Wu S, Yang YM, Zhu J, et al. Impact of baseline neutrophil-tolymphocyte ratio on long-term prognosis in patients with atrial fibrillation. Angiology. 2021;33197211000495. doi:10.1177/0003319 7211000495

36. Chen Y, Chang G, Chen X, et al. IL-6-miR-210 suppresses regulatory $\mathrm{T}$ cell function and promotes atrial fibrosis by targeting Foxp3. Mol Cells. 2020;43(5):438-447. doi:10.14348/molcells.2019.2275

37. Matsumoto K, Ogawa M, Suzuki J, Hirata Y, Nagai R, Isobe M. Regulatory $\mathrm{T}$ lymphocytes attenuate myocardial infarction-induced ventricular remodeling in mice. Int Heart J. 2011;52(6):382-387. doi:10.1536/ihj.52.382

38. Ong S, Rose NR, Čiháková D. Natural killer cells in inflammatory heart disease. Clin Immunol. 2017;175:26-33. doi:10.1016/j.clim.20 16.11.010

39. Backteman K, Ernerudh J, Jonasson L. Natural killer (NK) cell deficit in coronary artery disease: no aberrations in phenotype but sustained reduction of $\mathrm{NK}$ cells is associated with low-grade inflammation. Clin Exp Immunol. 2014;175(1):104-112. doi:10.1111/cei.12210

40. Backteman K, Andersson C, Dahlin LG, Ernerudh J, Jonasson L. Lymphocyte subpopulations in lymph nodes and peripheral blood: a comparison between patients with stable angina and acute coronary syndrome. PLoS One. 2012;7(3):e32691. doi:10.1371/journal.pone. 0032691

41. Tosello-Trampont A, Surette FA, Ewald SE, Hahn YS. Immunoregulatory role of NK cells in tissue inflammation and regeneration. Front Immunol. 2017;8:301. doi:10.3389/fimmu.2017.00301

42. Stellos K, Rahmann A, Kilias A, et al. Expression of platelet-bound stromal cell-derived factor-1 in patients with non-valvular atrial fibrillation and ischemic heart disease. J Thromb Haemost. 2012;10 (1):49-55. doi:10.1111/j.1538-7836.2011.04547.x

43. Cristillo AD, Bierer BE. Regulation of CXCR4 expression in human T lymphocytes by calcium and calcineurin. Mol Immunol. 2003;40 (8):539-553. doi:10.1016/S0161-5890(03)00169-X

44. Wu Q, Shao H, Darwin ED, et al. Extracellular calcium increases CXCR4 expression on bone marrow-derived cells and enhances pro-angiogenesis therapy. J Cell Mol Med. 2009;13(9b):3764-3773. doi:10.1111/j.1582-4934.2009.00691.x

45. Wang XX, Zhang FR, Zhu JH, Xie XD, Chen JZ. Up-regulation of $\mathrm{CXC}$ chemokine receptor 4 expression in chronic atrial fibrillation patients with mitral valve disease may be attenuated by renin-angiotensin system blockers. J Int Med Res. 2009;37 (4):1145-1151. doi:10.1177/147323000903700419

46. Liu P, Sun H, Zhou X, et al. CXCL12/CXCR4 axis as a key mediator in atrial fibrillation via bioinformatics analysis and functional identification. Cell Death Dis. 2021;12(9):813. doi:10.1038/s41419021-04109-5

47. Wu XM, Ji KQ, Wang HY, et al. MicroRNA-339-3p alleviates inflammation and edema and suppresses pulmonary microvascular endothelial cell apoptosis in mice with severe acute pancreatitis-associated acute lung injury by regulating Anxa3 via the Akt/mTOR signaling pathway. J Cell Biochem. 2018;119 (8):6704-6714. doi:10.1002/jcb.26859

48. Zhang D, Contu R, Latronico MV, et al. MTORC1 regulates cardiac function and myocyte survival through 4E-BP1 inhibition in mice. J Clin Invest. 2010;120(8):2805-2816. doi:10.1172/JCI43008 
49. Sciarretta S, Forte M, Frati G, Sadoshima J. New insights into the role of mTOR signaling in the cardiovascular system. Circ Res. 2018;122(3):489-505. doi:10.1161/CIRCRESAHA.117.311147

50. Daams R, Massoumi R. Nemo-like kinase in development and diseases: insights from mouse studies. Int J Mol Sci. 2020;21(23):23. doi:10.3390/ijms21239203

51. Liu R, Khalil H, Lin SJ, Sargent MA, York AJ, Molkentin JD. NemoLike Kinase (NLK) is a pathological signaling effector in the mouse heart. PLoS One. 2016;11(10):e0164897. doi:10.1371/journal.pone. 0164897

52. Alturaiki W. The roles of $B$ cell activation factor (BAFF) and a proliferation-inducing ligand (April) in allergic asthma. Immunol Lett. 2020;225:25-30. doi:10.1016/j.imlet.2020.06.001

53. Mo F, Luo Y, Yan Y, Li J, Lai S, Wu W. Are activated B cells involved in the process of myocardial fibrosis after acute myocardial infarction? An in vivo experiment. BMC Cardiovasc Disord. 2021;21 (1):5. doi:10.1186/s12872-020-01775-9
54. Huang Y, Chen L, Feng Z, et al. EPC-derived exosomal miR-1246 and miR-1290 regulate phenotypic changes of fibroblasts to endothelial cells to exert protective effects on myocardial infarction by targeting ELF5 and SP1. Front Cell Dev Biol. 2021;9:647763. doi:10.3389/fcell.2021.647763

55. Becher PM, Lindner D, Fröhlich M, Savvatis K, Westermann D, Tschöpe C. Assessment of cardiac inflammation and remodeling during the development of streptozotocin-induced diabetic cardiomyopathy in vivo: a time course analysis. Int J Mol Med. 2013;32 (1):158-164. doi:10.3892/ijmm.2013.1368

56. Moreno-Viedma V, Amor M, Sarabi A, et al. Common dysregulated pathways in obese adipose tissue and atherosclerosis. Cardiovasc Diabetol. 2016;15(1):120. doi:10.1186/s12933-016-0441-2

\section{Publish your work in this journal}

The International Journal of General Medicine is an international, peer-reviewed open-access journal that focuses on general and internal medicine, pathogenesis, epidemiology, diagnosis, monitoring and treatment protocols. The journal is characterized by the rapid reporting of reviews, original research and clinical studies across all disease areas. The manuscript management system is completely online and includes a very quick and fair peer-review system, which is all easy to use. Visit http://www.dovepress.com/ testimonials.php to read real quotes from published authors. 